\title{
Towards non-perturbative matching of three/four-flavor Wilson coefficients with a position-space procedure
}

\author{
Masaaki Tomii* \\ Physics Department, Columbia University, New York 10027, USA \\ E-mail: mt3164acolumbia.edu
}

\begin{abstract}
We propose a strategy to non-perturbatively match the Wilson coefficients in the three- and fourflavor theories, which uses two-point Green's functions of the corresponding four-quark operators at long distances. The idea is refined by combining with the spherical averaging technique, which enables us to convert two-point functions calculated on the lattice into continuous functions of the distance $|x-y|$ between two operators. We also show the result for an exploratory calculation of two-point functions of the $\Delta S=1$ operators $Q_{7}$ and $Q_{8}$ that are in the $\left(8_{L}, 8_{R}\right)$ representation of $\mathrm{SU}(3)_{L} \times \mathrm{SU}(3)_{R}$ and mix with each other.
\end{abstract}

The 36th Annual International Symposium on Lattice Field Theory - LATTICE2018

22-28 July, 2018

Michigan State University, East Lansing, Michigan, USA.

${ }^{*}$ Speaker. 


\section{Introduction}

Lattice calculations of weak matrix elements play an important role in searching for physics beyond the Standard Model. Weak-boson exchanges in low-energy processes can be reduced to an effective weak Hamiltonian composed of four-quark operators by integrating out the weak bosons and quarks heavier than the renormalization scale $\mu$. Then, the information at high energies $>\mu$ is expressed in terms of the Wilson coefficients, the coefficients of the four-quark operators in the weak Hamiltonian.

For many processes, the corresponding Wilson coefficients are known to one- or two-loop level in perturbative QCD both in the $\overline{\mathrm{MS}}$ and $\mathrm{RI} /(\mathrm{S}) \mathrm{MOM}$ schemes. Therefore the four-quark operators need to be renormalized at the same renormalization scale and in the same scheme as the Wilson coefficients to construct the proper weak Hamiltonian. The RI/(S)MOM scheme is more straightforward than the $\overline{\mathrm{MS}}$ scheme for actual lattice calculations.

We also need to match the number of flavors in the renormalization scheme of the Wilson coefficients and the four-quark operators. The perturbative calculation of the Wilson coefficients in the three-flavor theory needs a conversion from those in the four-flavor theory at an energy scale below the charm threshold $m_{c} \simeq 1.3 \mathrm{GeV}$, where perturbative calculation is quite ambiguous. While the difference between the three- and four-flavor Wilson coefficients is not significant if the form of the four-quark operators in the three- and four-flavor theories is the same and the sea charm effect is not significant, the issue is more serious when the charm quark can be involved in the four-quark operators in the four-flavor theory. In such a case, it is preferable to introduce the fourquark operators in the four- or five-flavor theory so that we do not need the three-flavor Wilson coefficients. However, if the lattice ensemble on which matrix elements are calculated is too coarse $a^{-1} \leq 2 \mathrm{GeV}$ to introduce the charm quark, a non-perturbative matching of the Wilson coefficients between the three- and four-flavor theories is needed. The RBC and UKQCD collaborations are facing this issue in the calculation of direct CP-violating effects in $K \rightarrow \pi \pi$ decays. Their original result contained $12 \%$ systematic uncertainty because of the perturbative matching of the Wilson coefficients Ref. [四].

In this work, we formulate a strategy to nonperturbatively match the three- and four-flavor Wilson coefficients and perform some exploratory calculations. As explained in Section $\square$, the strategy basically uses the two-point functions of four-quark operators, which are gauge invariant and prevent mixing with gauge-noninvariant operators and operators that are forbidden by equations of motion. In order to take the continuum limit of the matching matrix accurately, we propose to take the spherical average of two-point functions [2], which is briefly explained in Section B]. Some exploratory results for the spherical average of two-point functions of the three-flavor operators in the $\left(8_{\mathrm{L}}, 8_{\mathrm{R}}\right)$ representation are shown in Section 4 .

\section{Non-perturbative three/four-flavor matching of Wilson coefficients}

We start with the weak Hamiltonian

$$
H_{W}=\left.\sum_{i} w_{n_{f}, i}^{S_{n_{f}^{\prime}}}(\mu) O_{n_{f}, i}^{S_{n_{f}^{\prime}}}(\mu)\right|_{n_{f}^{\prime}=n_{f}}=\left.w_{n_{f}}^{S_{n_{f}^{\prime}}}(\mu)^{T} O_{n_{f}}^{S_{n_{f}^{\prime}}}(\mu)\right|_{n_{f}^{\prime}=n_{f}},
$$


where $\mu$ denotes the renormalization scale in a scheme indicated by the superscript $S_{n_{f}^{\prime}}$. The number of flavors $n_{f}^{\prime}$ as the subscript of $S$ is the number of sea quarks, while the $n_{f}$ as the subscript of $O$ and $w$ is the number of valence quarks that characterizes the concrete form of operators. For simplicity, we use vector and matrix notation as in the RHS of Eq. (ل्.] by omitting the index $i$ of operators. The superscript $T$ denotes the transposition of the vector or matrix. The weak Hamiltonian is independent of $n_{f}$ in the sense that matrix elements calculated in QCD between states which involve an energy scale $E$ do not change when $n_{f}$ is increased above $n_{f}^{\mathrm{e} f f}$, which is chosen so that quark flavors indexed by $n>n_{f}^{\mathrm{e} f f}$ have masses $m \gg E$.

If we calculate weak matrix elements with three-flavor operators in 2+1-flavor QCD ensembles, we need the Wilson coefficients $w_{3}^{S_{3}}(\mu)$ in the three-flavor theory to obtain the proper weak Hamiltonian

$$
\left\langle\pi \pi\left|H_{W}\right| K\right\rangle=w_{3}^{S_{3}}(\mu)^{T}\left\langle\pi \pi\left|O_{3}^{S_{3}}(\mu)\right| K\right\rangle_{2+1} .
$$

However, perturbative calculation of $w_{3}^{S_{3}}(\mu)$ requires a matching from $w_{4}^{S_{4}}\left(\mu^{\prime}\right)$ that is performed below the charm threshold, which induces a large systematic error $(\sim 12 \%)$ [W]. Therefore a nonperturbative matching in a non-perturbative scheme is desired. The RI/(S)MOM scheme is not suitable since it cannot prevent mixing with irrelevant operators allowed by a gauge-fixing and contact terms, which may become more important at low scales. A position-space scheme $X$ is a reasonable scheme to implement the non-perturbative matching since it prevents significant mixing with gauge-noninvariant operators and operators that are forbidden by the equations of motion.

We consider the equality of two-point function $\left\langle H_{W}(x) O_{3}^{X_{n_{f}}}(\mu ; y)^{\dagger}\right\rangle_{N_{f}}$ for 3 and 4 flavors:

$$
w_{3}^{S_{3}}\left(\mu_{3}\right)^{T}\left\langle O_{3}^{S_{3}}\left(\mu_{3} ; x\right) O_{3}^{X_{3}}(\mu ; y)^{\dagger}\right\rangle_{2+1}=w_{4}^{S_{4}^{\prime}}\left(\mu_{4}\right)^{T}\left\langle O_{4}^{S_{4}^{\prime}}\left(\mu_{4} ; x\right) O_{3}^{X_{4}}(\mu ; y)^{\dagger}\right\rangle_{2+1+1},
$$

which is valid at long distances $1 /|x-y| \ll m_{c}$. Then we obtain

$$
\begin{aligned}
w_{3}^{S_{3}}\left(\mu_{3}\right)=\left(Z_{O_{3}}^{S_{3} / \text { lat }}\left(\mu_{3}, 1 / a\right)^{T}\right)^{-1} G_{3-3}^{\mathrm{lat}[2+1]}(1 / a ; x-y)^{-1} Z_{O_{3}}^{X_{3} / \mathrm{lat}}(\mu, 1 / a)^{-1} \\
\quad \times Z_{O_{3}}^{X_{4} / \mathrm{lat}}(\mu, 1 / a) G_{3-4}^{\mathrm{lat}[2+1+1]}(1 / a ; x-y) Z_{O_{4}}^{S_{4}^{\prime} / \mathrm{lat}}\left(\mu_{4}, 1 / a\right)^{T} w_{4}^{S_{4}^{\prime}}\left(\mu_{4}\right),
\end{aligned}
$$

where we define

$$
G_{n_{f}-n_{f}^{\prime}}^{\mathrm{lat}\left[\mathrm{N}_{\mathrm{f}}\right]}(1 / a ; x-y)=\left\langle O_{n_{f}}^{\mathrm{lat}}(1 / a ; x) O_{n_{f}^{\prime}}^{\mathrm{lat}}(1 / a ; y)^{\dagger}\right\rangle_{N_{f}},
$$

and introduce renormalization matrices which satisfy

$$
O_{n_{f}}^{S_{n_{f}^{\prime}}}(\mu ; x)=Z_{O_{n_{f}}}^{S_{n_{f}^{\prime}} / \text { lat }}(\mu, 1 / a) O_{n_{f}}^{\text {lat }}(1 / a ; x) .
$$

If the sea charm quark is neglected, the relation becomes easier

$$
\begin{aligned}
w_{3}^{S_{3}}\left(\mu_{3}\right) \simeq\left(Z_{O_{3}}^{S_{3} / \mathrm{lat}}\left(\mu_{3}, 1 / a\right)^{T}\right)^{-1} & G_{3-3}^{\mathrm{lat}[2+1]}(1 / a ; x-y)^{-1} \\
& \times G_{3-4}^{\mathrm{lat}[2+1]}(1 / a ; x-y) Z_{O_{4}}^{S_{3}^{\prime} / \mathrm{lat}}\left(\mu_{4}, 1 / a\right)^{T} w_{4}^{S_{4}^{\prime}}\left(\mu_{4}\right),
\end{aligned}
$$


so that renormalization matrices in the position-space scheme are not needed. In addition, if the three-flavor operators are the same as the four-flavor operators $O_{3}=O_{4}$, i.e. if the valence charm quark cannot be involved in the operators, the matching between the three- and four-flavor Wilson coefficients is identical as long as the sea charm quark is neglected. On the other hand, if the charm quark is present in the four-flavor operators, as in the case of $K \rightarrow \pi \pi$ decays, the matching of the Wilson coefficients is needed if the weak matrix elements are to be calculated with the three-flavor operators. Note that we actually need the lattice Wilson coefficients $w_{3}^{\text {lat }}$, which can be obtained from Eq. (L.]) if we simply drop the multiplication by $\left(Z_{O_{3}}^{S_{3} / \text { lat }}\right)^{-1}$ from the RHS of Eq. ([2.]), removing any reference to the scheme $S_{3}$.

We will choose $S=S^{\prime}=\mathrm{RI} / \mathrm{SMOM}$, in which the Wilson coefficients in the four-flavor theory can be calculated perturbatively. To obtain the Wilson coefficients in the three-flavor theory, we need to calculate the four-flavor renormalization matrix $Z_{O_{4}}^{\mathrm{RI} / \mathrm{SMOM}_{3} / \text { lat }}(\mu, 1 / a)$, the two-point Green's functions of two three-flavor operators $G_{3-3}^{\text {lat }[2+1]}(1 / a ; x-y)$ and those between a threeflavor operator and a four-flavor operator $G_{3-4}^{\mathrm{lat}[2+1]}(1 / a ; x-y)$. In the following sections, we fix $y=0$ for simplicity and present our strategy to calculate the two-point functions with controlled discretization errors and the results from a test calculation.

\section{Spherical average of two-point functions}

The three-flavor Wilson coefficients calculated with the strategy proposed in the previous section depend on $x$, the relative distance between two operators in the correlators. As long as we investigate in the region $1 /|x| \ll m_{c}$, the $x$-dependence may show some unphysical behavior and the finite cutoff effects may be a possible source of it. Although distance scale in $1 /|x| \ll m_{c}$ is much longer than recently used lattice spacings, discretization effects on correlators at $1 /|x| \simeq 400 \mathrm{MeV}$ are more than $10 \%$ depending on lattice spacing and much larger than statistical errors. Thus, it is preferable to take the continuum limit to avoid such ambiguity. However, in order to take the continuum limit, we need to calculate correlators at a fixed physical distance for each lattice spacing, while correlators on the lattice have values only at discrete points that depend on lattice spacing.

We will apply the spherical averaging technique [వ] to evaluate correlators at any physical distance as well as to reduce discretization errors. While correlators on the lattice violate $O(4)$ symmetry and depend on lattice points in a complicated way, this technique enables us to obtain correlators that depend only on the distance $|x|$ as if they have $O(4)$ symmetry. There are two steps to evaluate sphere-averaged correlators using lattice correlators $f_{a, n}{ }^{1}$ :

- Interpolation In this step, we estimate the values of correlators at any physical location $x$. In the case of one dimension, it is easy to verify that the linear interpolation

$$
\bar{f}_{a}(x)=\frac{(a(n+1)-x) f_{a, n}+(x-a n) f_{a, n+1}}{a},
$$

\footnotetext{
${ }^{1}$ In Ref. []], we defined $f_{a, n}$ as a correlator multiplied by $x^{2 d}$ with the dimension $d$ of the operator and divided by the same factor after taking the spherical average to avoid strong $x$-dependence, which may induce a large discretization error as a by-product of the spherical average.
} 
cancels the $O\left(a^{1}\right)$ discretization error that arises from the Taylor expansions of $f_{a, n}$ and $f_{a, n}$ around $x$. In the case of four dimensions, the interpolation is modified to

$$
\bar{f}_{a}(x)=a^{-4} \sum_{i, j, k, l=0}^{1} \Delta_{1, i} \Delta_{2, j} \Delta_{3, k} \Delta_{4, l} f_{a, n+i \hat{1}+j \hat{2}+k \hat{3}+l \hat{4}}
$$

where $n_{\mu}=\left\lfloor x_{\mu} / a\right\rfloor, \hat{\mu}$ is the unit vector for the $\mu$-direction and we define

$$
\Delta_{\mu, i}=\left|a\left(n_{\mu}+1-i\right)-x_{\mu}\right| .
$$

It is also easy to verify that this interpolation is free from $O\left(a^{1}\right)$ errors.

- Average over spheres While the interpolated correlators in four-dimensions designed above have values at any physical location, they still violate rotational symmetry and depend on $x$ in a complicated way. To obtain correlators as a continuous function of only the distance $|x|$, we average them over a four-dimensional sphere $U_{|x|}$ with the radius of $|x|$,

$$
\hat{f}_{a}(|x|)=\oint_{U_{|x|}} \mathrm{d} \sigma \bar{f}_{a}(x) / \oint_{U_{|x|}} \mathrm{d} \sigma .
$$

\section{Exploratory calculation of two-point functions of four-quark operators}

In this section, we show the result for a preliminary calculation of two-point functions

$$
G_{i j}(x)=\left\langle Q_{i}(x) Q_{j}(y)^{\dagger}\right\rangle
$$

of unrenormalized $\Delta S=1$ four-quark operators $Q_{i, j}$ in the three-flavor theory. In general, the calculation of these two-point functions requires all-to-all quark propagators since there are diagrams that contain a quark loop at the sink point. Thus, there may be power divergence from loop diagrams, which needs to be eliminated before renormalizing the operators.

Among the $\Delta S=1$ operators relevant for the $K \rightarrow \pi \pi$ matrix elements,

$$
\begin{aligned}
Q_{7} & =\frac{3}{2} \bar{s}_{\alpha} \gamma_{\mu}\left(1-\gamma_{5}\right) d_{\alpha} \sum_{q=u, d, s} e_{q} \bar{q}_{\beta} \gamma_{\mu}\left(1+\gamma_{5}\right) q_{\beta}, \\
Q_{8} & =\frac{3}{2} \bar{s}_{\alpha} \gamma_{\mu}\left(1-\gamma_{5}\right) d_{\beta} \sum_{q=u, d, s} e_{q} \bar{q}_{\beta} \gamma_{\mu}\left(1+\gamma_{5}\right) q_{\alpha},
\end{aligned}
$$

where $e_{q}$ is the electric charge of a quark $q$ the RHSs are summed over the Lorentz index $\mu$ and the color indices $\alpha$ and $\beta$, enable us to investigate the simplest case of mixing correlator matrix since only these two operators belong to the $\left(8_{L}, 8_{R}\right)$ representation of $\mathrm{SU}(3)_{L} \times \mathrm{SU}(3)_{R}$ symmetry [3], 田]. In this article, we show the result for the contribution of the fully-connected diagrams in which all the quark propagators connect the source and sink points and there is no power divergence. (These are the only non-zero diagrams if we use the $I=3 / 2$ components of $O_{7}$ and $O_{8}$.)

We use $2+1$-flavor domain-wall ensembles with three lattice cutoffs $a^{-1}$ ranging from $1.79 \mathrm{GeV}$ to $3.15 \mathrm{GeV}$ generated by the RBC and UKQCD collaborations. Pion masses are in the region from 

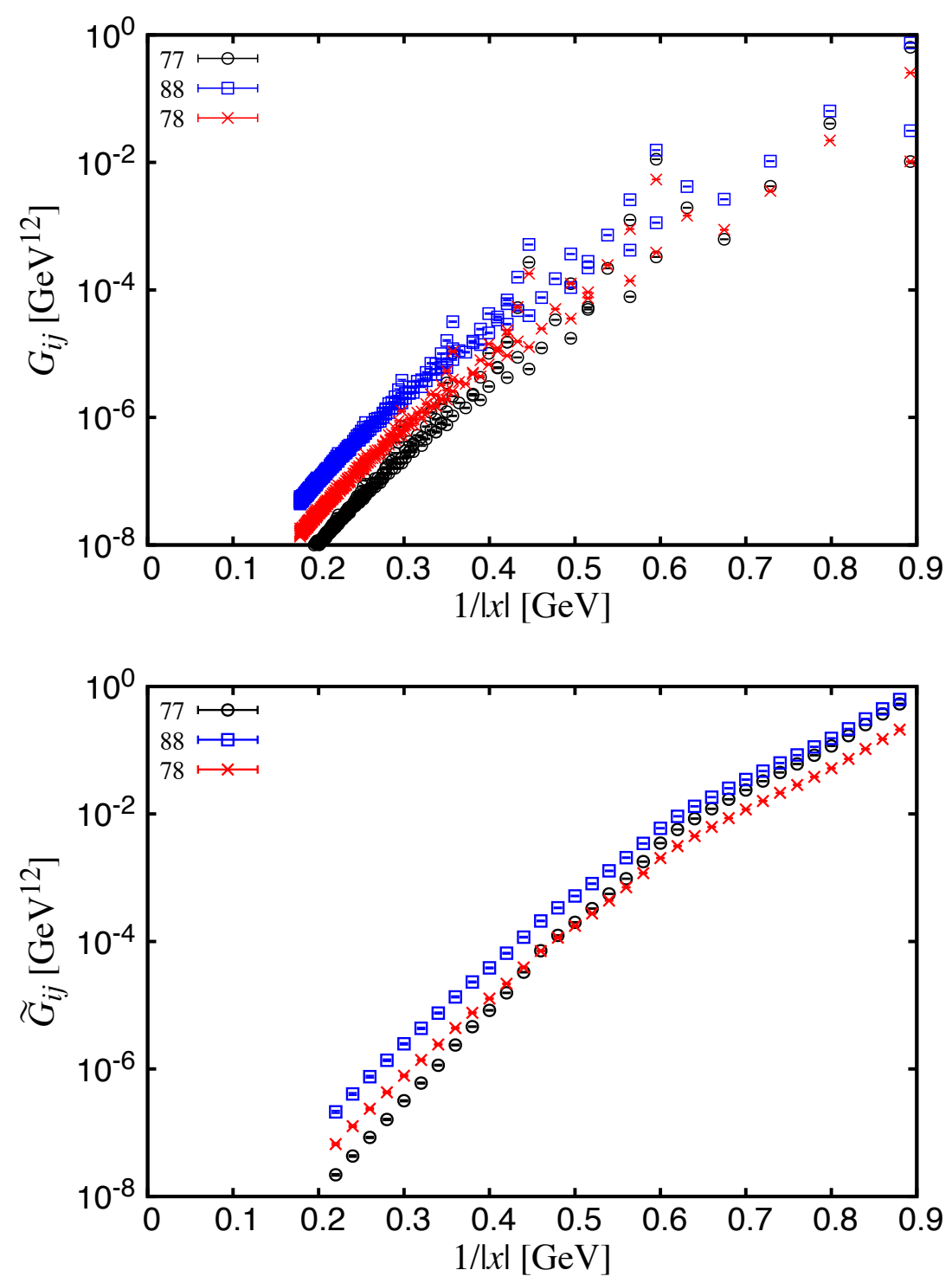

Figure 1: Results for $G_{77}(x)$ (circles), $G_{88}(x)$ (squares) and $G_{78}(x)$ (crosses) calculated on the coarsest lattice with $a^{-1} \simeq 1.79$ before (upper panel) and after (lower panel) taking the spherical average.

\section{$300 \mathrm{MeV}$ to $370 \mathrm{MeV}$.}

Figure $\mathbb{W}$ shows the results for two-point functions of $\left(8_{L}, 8_{R}\right)$ operators. Since the correlator matrix is real symmetric, we take the average of the 78 and 87 elements, which is shown as the 78 element (crosses) in the figure. The upper panel shows the results for $G_{i j}(x)$ before taking the spherical average. Here, we distinguish different lattice points that are not equivalent with respect to $90^{\circ}$ rotations or parity inversion in the four-dimensional hypercubic group. The results are averaged over sets of lattice points related by hypercubic transformations. The ambiguity due to the violation of $O(4)$ symmetry could amount to more than $\times 10$ at $|x|=3 a$ as numerical results at $|x|=3 a$ read $G_{77}(0,0,0,3 a)=1.12(1) \times 10^{-2} \mathrm{GeV}^{12}$ and $G_{77}(0, a, 2 a, 2 a)=3.41(3) \times 10^{-4} \mathrm{GeV}^{12}$. From the 

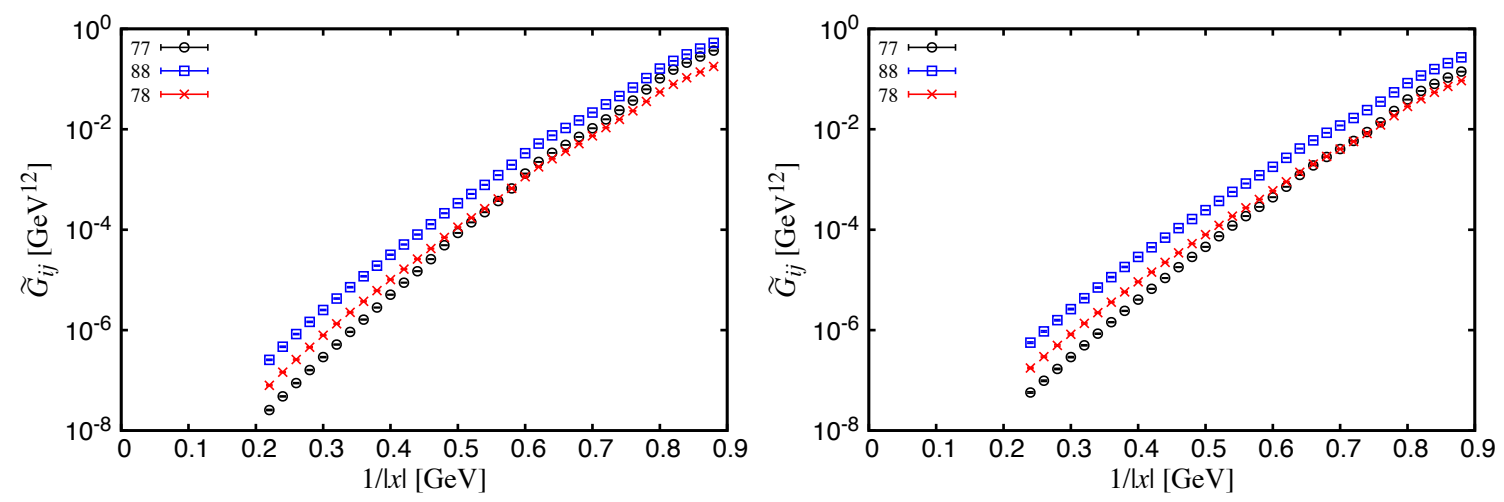

Figure 2: Same as the lower panel of Figure $\square$ but the result on finer lattices, $a^{-1}=2.38 \mathrm{GeV}$ (left panel) and $a^{-1}=3.15 \mathrm{GeV}$ (right panel).

same observation, the ambiguity at $|x|=6 a$ is about $\times 3$. The lower panel shows the results for the spherical average $\widetilde{G}_{i j}(|x|)$. The discretization errors in the spherical average appear to be much smaller than those in $G_{i j}(x)$. Figure $\square$ show the results for the the spherical average calculated on finer lattices, $a^{-1}=2.38 \mathrm{GeV}$ (left panel) and $a^{-1}=3.15 \mathrm{GeV}$ (right panel). As mentioned in the previous section, the spherical averaging technique enables us to evaluate the values of correlators at any physical distance. Therefore, the matching matrix between the Wilson coefficients in the three- and four-flavor theories Eq. (더) or (L.]), which is calculated from two-point functions, can easily be extrapolated to the continuum limit at any physical distance $|x|$.

\section{Summary}

We formulate a non-perturbative strategy to match the three- and four-flavor Wilson coefficients of $\Delta S=1$ four-quark operators. We propose to use two-point Green's functions of fourquark operators and their spherical average to take the continuum limit of the matching matrix. As Eq. (‥7) indicates, we also needs the renormalization matrix of the four-flavor operators in a scheme in which perturbative calculation is available. The four-flavor operators will be calculated as well as the two-point functions in near future.

\section{References}

[1] Z. Bai et al. [RBC and UKQCD Collaborations], Phys. Rev. Lett. 115, no. 21, 212001 (2015) doi:10.1103/PhysRevLett.115.212001 [arXiv:1505.07863 [hep-lat]].

[2] M. Tomii and N. H. Christ, arXiv:1811.11238 [hep-lat].

[3] T. Blum et al. [RBC Collaboration], Phys. Rev. D 68, 114506 (2003) doi:10.1103/PhysRevD.68.114506 [hep-lat/0110075].

[4] C. Bernard, Lectures given at TASI'89, Boulder, CO, 1989 